\title{
Internal Structure of Beck Hopelessness Scale: An Analysis of Method Effects Using the CT-C(M-1) Model
}

\author{
Pablo Ezequiel Flores-Kanter, Ronald Toro \& Jesús M. Alvarado
}

Accepted for publication at Journal of Personality Assessment on May 21, 2021

(C) 2021 Taylor \& Francis Group, LLC. This paper is not the copy of record and may not exactly replicate the final, authoritative version of the article. Please do not copy or cite without authors' permission.

To link to this article: https://doi.org/10.1080/00223891.2021.1942021

To cite this article: Flores-Kanter, P. E., Toro, R., \& Alvarado, J. M. (2022). Internal structure of Beck Hopelessness Scale: An analysis of method effects using the CT-C (M-1) model. Journal of Personality Assessment, 104(3), 408-416. https://doi.org/10.1080/00223891.2021.1942021 


\title{
Internal Structure of Beck Hopelessness Scale: An Analysis of Method Effects Using the CT-C $(M-1)$ Model
}

\author{
Pablo Ezequiel Flores-Kanter ${ }^{1,2,3}$, Ronald Toro ${ }^{4}$, and Jesús M. Alvarado 5 \\ ${ }^{1}$ National Scientific and Technical Research Council (CONICET), Consejo Nacional de Investigaciones Científicas y Técnicas, CONICET, \\ Argentina; ${ }^{2}$ Instituto Tecnológico de Buenos Aires; ${ }^{3}$ Centre for Bioethics, Catholic University of Cordoba (UCC), Universidad Católica de \\ Córdoba, UCC, Argentina; ${ }^{4}$ Psychology Department, Catholic University of Colombia; ${ }^{5}$ Faculty of Psychology, Psychobiology \& Behavioral \\ Sciences Methods Department, Complutense University of Madrid
}

\begin{abstract}
ABTRACT
The construct validity in relation to the dimensionality or factor structure of the Beck Hopelessness Scale (BHS) has long been debated in psychometrics. Irrelevant variance due to item wording (method effects) can distort the factor structure, and recent studies have examined the method factor's role in the factor structure of the BHS. However, the models used to control the method effects have severe limitations, and new models are needed. One such model is the correlated trait-correlated method minus one (CT-C(M-1)), which is a powerful approach that gives the trait factor an unambiguous meaning and prevents the anomalous results associated with fully symmetrical bifactor modeling. The present work compares the fit and factor structure of the CT$\mathrm{C}(\mathrm{M}-1)$ model to bifactor models proposed in previous literature and evaluates the criterion validity of the CT-C(M-1) model and its discriminatory capacity by taking suicidal ideation as the criterion variable. This study used a large and heterogeneous open mode online sample of Argentinian people $(N=2,164)$. The results indicated that the CT-C $(M-1)$ model with positive words as referenced items achieves the most adequate factor structure. The factorial scores derived from this model demonstrate good predictive and discriminating capabilities.
\end{abstract}

\section{Introduction}

The Beck Hopelessness Scale (BHS) is a self-assessment questionnaire designed to measure negative attitudes toward the future (Beck et al., 1974). The BHS has become one of the most popular measurements of the hopelessness construct in international studies (Kliem et al., 2018). One common practice in previous BHS research is, after recoding negatively worded items, to combine the number of endorsed items into a sum-score (Brown et al., 2000; FloresKanter et al., 2019; Gran6 et al., 2017; Serafini et al., 2020; Tsujii et al., 2020).

The use of the total BHS score assumes that the psychometric evidence favors a one-dimensional model. However, the factor structure of the scale has been extensively debated, and different internal structure solutions have been suggested for the BHS (Boduszek \& Dhingra, 2016; Kocalevent et al., 2017). The three-factor model (Beck et al., 1974) (i.e., feelings about the future, loss of motivation, future expectations) and the one-dimensional model (Aish \& Wasserman, 2001) are commonly discussed in the literature. More recently, the influence of method factors (MFs) has been pointed out (i.e., optimism and pessimism in item wording), as discussed by Boduszek and Dhingra (2016), Innamorati et al. (2014), Kliem et al. (2018), and Szabó et al. (2016). In their research, bifactor models were used to consider specific factors concerning the negative and positive wording of items, as well as a general orthogonal factor of hopelessness. The inconsistencies between different factor solutions and the lack of clarity of items that make up the factors impede the clear interpretation of BHS scores. This issue is relevant to the applied field because the common use of the raw or total BHS score in predictive studies may be based on a non-opti-

mal factor solution. More importantly, the use of these scores may increase the prevalence of systematic variance that is irrelevant to the measurement of the hopelessness construct. These aspects produce noise in the metrics obtained, which can lead to biased estimates and a loss of predictive capability. Several reasons may explain the significant divergence in the available evidence on the internal structure of the BHS. Many of these have been clearly identified by Boduszek and Dhingra (2016) and Szabó et al. (2016) and include the heterogeneity in the included samples (clinical vs. general population) and the application of non-conforming estimation methods for the BHS binary response format items that stand out. Beyond these possibilities, another relevant factor to consider is the format in which the items are written or the method effect of the item wording. 
Incorporation of negative items in the development of psychometric instruments

In many psychometric instruments, the incorporation of negative items is not due to the generation of a differential factor for a given construct. On the contrary, incorporation of reagents controls response bias, namely acquiescence bias (Savalei \& Falk, 2014). However, the incorporation of reversed or negative items in psychometric scales has been widely discussed (see Zhang \& Savalei, 2016).

In BHS factor studies reporting more than one factor, the resulting factor structure mainly separated the positively worded items from the negatively worded ones. Positively worded items tended to load on one factor, while negatively worded items tended toward multiple factors. Thus, it is possible to hypothesize that the generation of multi-factor structures in the BHS is a methodological artifact that results from the incorporation of negative items and does not reflect a substantive factor. Identifying the wording of items as dependent factors reflects the tendency of participants to respond in distinct ways to positively and negatively worded items, regardless of the intended content. This tendency yields a systematic variance that is irrelevant to the context of the study (DiStefano \& Motl, 2006) and causes biases in the covariance structure of the data (Tomas \& Oliver, 1999; Zhang \& Savalei, 2016).

Understanding this response bias may help reconcile the mixed reported pattern of the internal structure of the BHS and allow for more conclusive results among applied researchers. The method effect was recently considered in studies of the BHS factor structure (Boduszek \& Dhingra, 2016; Innamorati et al., 2014; Kliem et al., 2018; Szabó et al., 2016). In these studies, bifactor models were used to examine the specific factors involved in negative and positive item wordings and a general orthogonal factor of hopelessness. The findings of such studies show an adequate fit for these measurement models. However, these CT-calculometry (CT-CM)-based approaches (i.e., fully symmetrical bifactor modeling) have serious limitations, and their use for method effect control is questionable.

Geiser et al. (2008) have identified two major disadvantages of the CT-CM model: i) it is prone to identification problems, and ii) it often produces solutions with out-of-range parameter estimates. Moreover, the interpretation of the trait and method factors in the CT-CM model can be ambiguous (Heinrich et al., 2020). The correlated trait-correlated method minus one (CT-C (M-1); Eid, 2000) is a recently proposed approach aimed at overcoming these limitations.

The CT-C (M-1) is an approach that has yet to be considered in the study of the BHS's internal structure. The CT$\mathrm{C}(\mathrm{M}-1)$ model is a particular variant of the CT-CM, but the number of MFs is one fewer than the number of meth- ods included. The M-1 measure model is a powerful approach that gives the trait factor an unambiguous mean- ing and prevents the anomalous results associated with fully symmetrical bifactor modeling (Eid, 2020; Heinrich et al., 2020). The advantage of this model over the traditional CTCM models is that its trait, method, and error components are uncorrelated. This feature allows for the decomposing of variances and covariances of the nonreference measures into variance components, due to the influences of the trait, method, and measurement errors. Thus, M-1 models allow the separation of substantive content from the mechanism or method used to gain the responses (e.g., item wordings; Geiser et al., 2008). In this confirmatory factor analysis (CFA) approach, the first-order CFA model is taken as a starting point. One of the first-order factors is selected as a reference domain that ultimately defines the trait factor (i.e., it reflects the trait assessed by the reference domain and therefore has an unambiguous meaning). The MFs reflect the parts of a domain that the reference domain cannot predict (i.e., residual factors). In the case of the BHS, the items would be determined by a trait factor of pessimism-hopelessness and one MF (i.e., negative or reversed items: the "optimism" or positively worded items in the BHS). Thus, in this case, the M-1 model eliminates one of the suggested BHS MFs (see Figure 1). Negatively worded items (i.e., positive or direct items) are selected as the reference method or trait factor so that the model contains a nonreference (method) factor for the positively worded items.

Given the advantages of applying the M-1 model, we hypothesize that this model will produce better fit indexes than those observed for previous measurement models tested on the BHS (H1).

\section{Need for criterion-related validity}

The CT-C(M-1) model must present evidence of criterion validity. For this purpose, a semi-partial correlation approach by means of structural equation modeling (SEM) is suggested (Geiser et al., 2008). SEM can be considered a combination of factor analysis models (i.e., measurement models) and regression models (i.e., structural models) (Yuan \& Bentler, 2007); the latter can be used to statistically control for variables (i.e., partialling out). In other words, it allows for decomposing the variances and covariances of the measures into variance components due to trait, method, and measurement error influences. As indicated by Geiser et al. (2008), the lack of criterion validity is indicated by a large amount of methodspecific variance after statistically controlling for the trait factor. In the present research, we hypothesized that the trait factor that represents pessimism for its positive items strongly explains suicide ideation (SI) (H2). By contrast, the MFs would only have a small or statistically insignificant effect beyond the effect of the trait factor $(\mathrm{H} 3)$.

We also proposed validating the M-1 model by applying the receiver operating characteristic (ROC) curve procedures with high SI and non-SI groups. This was particularly useful in clarifying the discriminatory capacity of the factor scores obtained. Moreover, it allowed us to appreciate the relative advantage of using the factor score of the M-1 model over the raw total BHS score (i.e., BHS sum-score) in terms of discriminatory capacity. In the ROC analysis, the area under the curve (AUC) measures the performance of a classifier; a higher AUC value indicates a better classification. The AUC is particularly useful when diagnostic tests are being compared (Habibzadeh et al., 2016). We compared the discriminating ability of the M-1 factor score and BHS sum-score. 


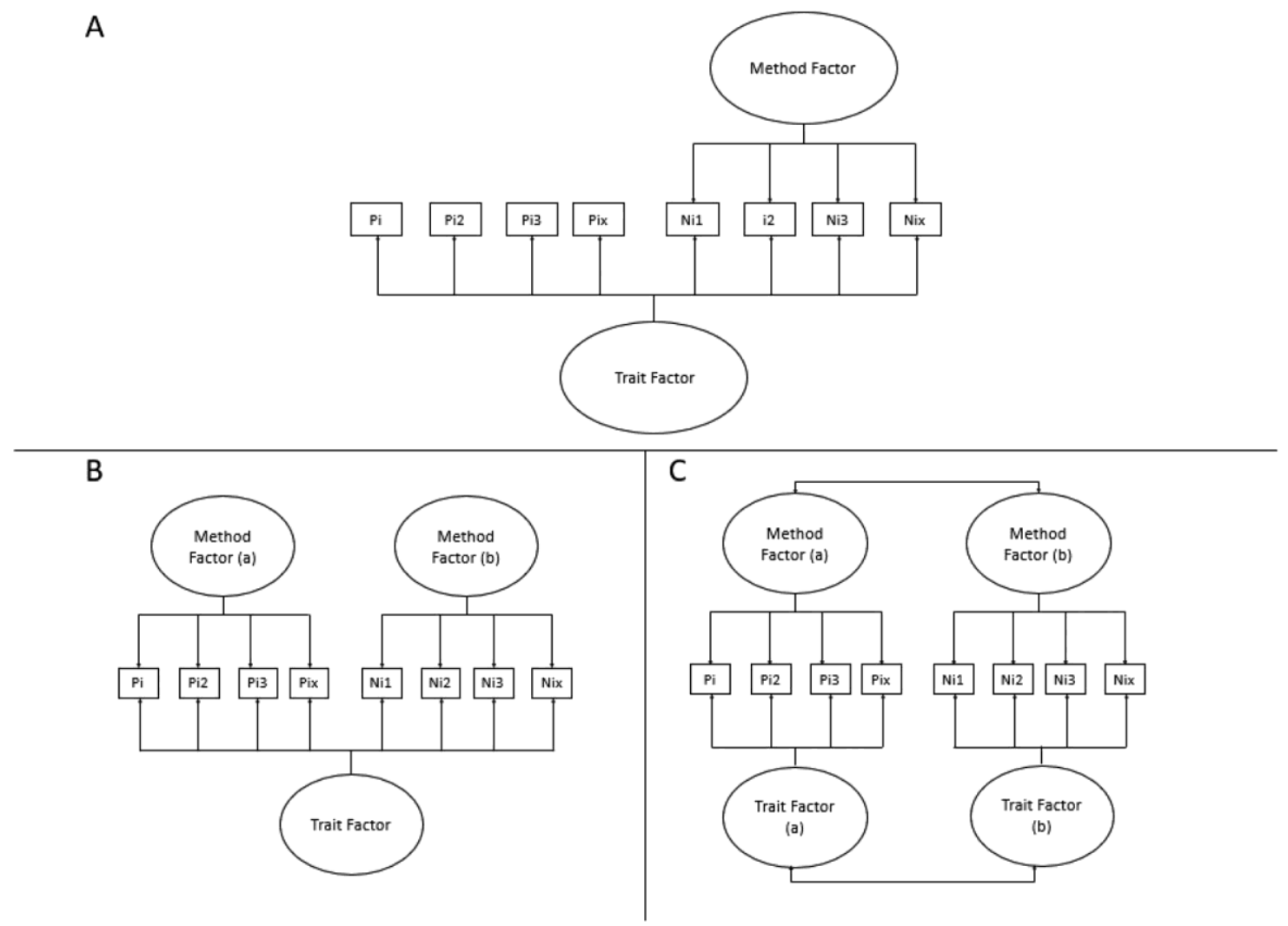

Figure 1. Examples of CT-C(M-1), bifactor, and MTMM models. $\mathrm{Pi}=$ positive items (negatively worded items in the Beck Hopelessness $\mathrm{Scale}(\mathrm{BHS}))$, Ni = negative items (positively worded items in the BHS). A: The correlated traits-correlated methods minus one model. B is a fully symmetrical bifactor model (Szabó et al., 2016) and $C$ is a multitrait-multimethod model (Boduszek \& Dhingra, 2016).

Note. CT-C(M-1), correlated trait-correlated method minus one; MTMM, multitrait-multimethod model.

Recent studies have verified that the AUC obtained by using the BHS sum-score for discrimination against SI groups varies within the range of .735 to .798 (Gran6 et al., 2017). We hypothesized that the discriminatory capacity of the M-1 factor score would be higher than observed in the previous literature, resulting in a statistically significant difference in the AUC values (H4). Given the common application of the BHS sum-score, obtaining evidence to confirm $\mathrm{H} 4$ allowed us to determine the practical significance of our findings.

\section{Objectives}

The main objective of this research was to verify the internal structure of the BHS in a large heterogeneous sample (in terms of gender, age, and clinical status). Unlike previous research, we applied a CT-C(M-1) model as a central objective. We also verified the criterion validity and the relative advantage of using the scores derived from the M-1 model in the prediction of SI.

\section{Materials and methods}

\section{Participants}

Participants were 2,164 Argentinians obtained through an open mode online sample method (The International Test Commission, 2006). This data collection methodology has proven to be equivalent to traditional forms of collection (i.e., face to face; Weigold et al., 2013), producing equal means, internal consistencies, intercorrelations, response rates, and comfort level when completing questionnaires. For this observational, cross-sectional study, data were collected using an online survey format to gather information through the Google Forms platform and were delivered by Facebook social media. The data were collected in November and December 2018. The sociodemographics of the participants are shown in Table 1.

All participants were adequately informed of the research objectives, the anonymity of their responses, and their voluntary participation. Likewise, it was clarified that participation would not cause any harm and that they could leave the study whenever they wished. International ethical guidelines for studies with human beings were considered (American Psychological Association, 2017). In this study, no specific incentive was used for participation in the study. In the case of minors under 18 years of age, prior parental consent was additionally requested. The ethics council of the Centre for Bioethics of the Catholic University of Cordoba previously approved the research protocol following APA ethical guidelines.

\section{Measures}

Beck hopelessness scale (BHS)

For this study, the adapted BHS version for the Argentinian population was used (Mikulic et al., 2009). This scale comprises 20 items with a dichotomous reply format (i.e., true 
Table 1. Demographic characteristics of sample.

\begin{tabular}{|c|c|c|}
\hline & $\mathrm{N}$ & $\%$ \\
\hline \multicolumn{3}{|l|}{ Gender } \\
\hline Female & 1,560 & 72.1 \\
\hline Male & 604 & 27.9 \\
\hline \multicolumn{3}{|l|}{ Age group } \\
\hline Young $(\leq 19)$ & 426 & 19.7 \\
\hline Aaulis (<U-5y) & ।,৩53 & 11.6 \\
\hline Older $(\leq 60)$ & 185 & 8.5 \\
\hline \multicolumn{3}{|l|}{ Argentina region } \\
\hline Northwest & 269 & 12.42 \\
\hline Pampean & 1,622 & 74.88 \\
\hline Cuyo & 136 & 6.28 \\
\hline Patagonia & 137 & 6.33 \\
\hline \multicolumn{3}{|l|}{ Actual treatment } \\
\hline No & 1,851 & 85.5 \\
\hline Yes & 313 & 14.5 \\
\hline \multicolumn{3}{|l|}{ Presence of SI } \\
\hline High & 82 & 3.8 \\
\hline Low-Moderate & 1,153 & 39.1 \\
\hline No SI & 1,235 & 57.1 \\
\hline \multicolumn{3}{|c|}{$\begin{array}{l}\text { Note. Actual treatment psychiatric or psychological. The presence of suicide } \\
\text { ideation (SI) was identified based on the recategorization of scores obtained } \\
\text { from the Inventory of Suicide Orientation-30 (ISO-30; range value 4-16). } \\
\text { No SI = a total score of } 4 \text { (i.e., the participant marked "strongly disagree" } \\
\text { on all the items); High = a score of } 16 \text { (i.e., the participant marked } \\
\text { "strongly agree" on all the items); Low-Moderate = correspond to } \\
\text { values between High and No SI. }\end{array}$} \\
\hline
\end{tabular}

or false) and is used to evaluate the respondent's negative expectations for the future. The instrument shows an adequate level of internal consistency $\left(a^{1 / 4} .78\right)$. In the present sample, an adequate Cronbach's alpha coefficient for the dimension was observed $(a=.88)$.

\section{Inventory of suicide orientation-30 (ISO-30)}

The version of the Inventory of Suicide Orientation-30 (ISO30) validated by Fernandez-Liporace and Casullo (2006) in Argentina was used for this study. The instrument measures the respondent's level of agreement with certain statements using a four-point Likert scale (with responses ranging from 1, "I strongly disagree" to 4, "I strongly agree"). From the inventory, only the questions that were related to the dimension of SI were included (e.g., "In order to stop things from getting worse, I believe suicide is the solution"). Within the ISO-30 scale, the suicidal ideation fac- tor is the one that has shown the highest consistency and evidence of measurement validity in the literature on the internal structure of the scale (Vecco et al., 2021). In the present sample, an adequate Cronbach's alpha coefficient for the dimension was observed $(a=.93)$.

\section{Statistical analyses}

First, CFA was applied. We tested the four most commonly discussed models in the BHS literature (see Boduszek \& Dhingra, 2016): (1) the original three-factor model by Beck et al. (1974; Model 1), comprising "feelings about the future" (items: 1, 5, 6, 13, 15, 19), "loss of motivation" (items: 2, 3, $9,11,12,16,17,20$ ), and "future expectations" (items: 4,7 , $8,10,14,18)$; (2) the one-dimensional model (all items loading on one overall-general factor; Model 2); (3) the bifactor model as suggested by Szabó et al. (2016; Model 3), with one general factor (all items) and two MFs (pessimistically worded items: $2,4,7,9,11,12,14,16,17$, 18, 20; and optimistically worded items: $1,3,5,6,8,10,13$, $15,19)$; and (4) the multitrait-multimethod model suggested by Boduszek and Dhingra (2016; Model 4), with three correlated trait factors (as suggested by Beck et al., 1974) and two correlated MFs. Finally, we added (5) the M-1 model proposed in the present research (Model 5).

Given the categorical nature of the observable variables in the BHS, the models were analyzed using the weighted least square mean and variance (WLSMV) estimator (Flora \& Curran, 2004; Rhemtulla et al., 2012). The overall fit of the models was evaluated using the root mean square error of approximation (RMSEA), the comparative fit index (CFI), and the Tucker-Lewis index (TLI). RMSEA values below .08 and .05 are considered indicative of reasonable and proper fit, respectively. CFI and TLI values above .90 and .95 reflect an acceptable and good adjustment, respectively ( $\mathrm{Hu} \&$ Bentler, 1999; Marsh et al., 2004). To compare the two given nested models, a T3 second-order correction for the chisquare difference testing method was used (Asparouhov \& Muthén, 2006). This method is appropriate when the WLSMV estimator is used. In addition, the quality of model fit was assessed based on the adequacy and plausibility of the estimated coefficients (Geiser et al., 2008; Heinrich et al., 2020).

The structural model proposed for the prediction of the SI was estimated using the same approach in the measurement model analysis (i.e., CFA). The criterion validity of the reference trait factor is supported if the variance explained by the MFs is smaller than the variance accounted for by the trait factor (Geiser et al., 2008). This was assessed from the standardized regression weights, the estimated correlation coefficients, and the statistical significance obtained for these parameters. Mplus (version 8) was used for CFA and SEM statistical analyses.

Finally, the ROC curves were analyzed, for which the AUC and their respective confidence intervals were calculated (G6nen, 2007). A ROC curve diagram indicates the performance of a binary classification method in terms of its sensitivity (i.e., correctly classified positive observations) and specificity (i.e., correctly classified negative observations). The ROC plot also displays the performance of a binary classification method with discrete ordinal output (Robin et al., 2011). In this research, the ranges of values obtained from the factor scores of the M-1 model were used as a starting point, verifying the performance of a SI group classification. Furthermore, the ROC curve and AUC analysis were based on an extreme case-control design. This type of design is applied to compare groups that lie at both extremes of a specific variable range. (Granat et al., 2017; Zhu et al., 2013). In the present research, the high-SI and noSI groups were identified based on the scores obtained from the ISO-30 SI subscale (possible range values 4/446). A total score of 4 (i.e., the participant marked "strongly disagree" on all items) was considered to indicate an absence of SI, while a score of 16 (i.e., the participant marked "strongly agree" on all items) indicated a high SI. Individuals scoring in the middle range were not included in the analyses (see 
Table 2. Fit indices of the models proposed for BHS.

\begin{tabular}{|c|c|c|c|c|c|c|c|}
\hline Model Fit & $v^{2}$ & df & CFI & TLI & RMSEA & SRMR & Anomalous Results \\
\hline Model 1 & 764.60 & 167 & .983 & .981 & .041 & .050 & NO \\
\hline Model 3 & 576.50 & 150 & .988 & .985 & .036 & .044 & YES \\
\hline Model 4 & 431.14 & 160 & .992 & .990 & .030 & .034 & YES \\
\hline Difference Testing & $v^{2}$ & df & $p$ & & & & \\
\hline Model 1 vs 5 & 51.13 & 6 & $<.001$ & & & & \\
\hline Model 2 vs 5 & 169.66 & 9 & $<.001$ & & & & \\
\hline
\end{tabular}

Note. BHS, Beck Hopelessness Scale. Model 1 the original three-factor model by Beck et al. (1974); Model 2 the one-dimensional model; Model 3 the bifactor model, as suggested by Szabó et al. (2016); Model 4 the multitrait-multimethod model suggested by Boduszek and Dhingra (2016); Model 5 the correlated trait-correlated method minus one (CT-C(M-1)) model.

Table 3. Standardized factor loadings for CT-C(M-1) model.

\begin{tabular}{|c|c|c|c|c|}
\hline & Estimate & $S E$ & Estimate/SE & $p$ \\
\hline \multicolumn{5}{|l|}{ Trait Factor } \\
\hline BHS1 & -.795 & .017 & 46.802 & $<.001$ \\
\hline BHŞ2 & $\begin{array}{r}.885 \\
-.679\end{array}$ & .015 & $\begin{array}{r}-58.702 \\
27.790\end{array}$ & $<.001$ \\
\hline BHS4 & .545 & .026 & -21.351 & $<.001$ \\
\hline BHS5 & -.554 & .025 & 22.502 & $<.001$ \\
\hline BHS6 & -.637 & .034 & 18.992 & $<.001$ \\
\hline BHS7 & .888 & .012 & -76.749 & $<.001$ \\
\hline BHS8 & -.697 & .02 & 35.680 & $<.001$ \\
\hline BHS9 & .882 & .013 & -67.318 & $<.001$ \\
\hline BHS10 & -.646 & .023 & 27.910 & $<.001$ \\
\hline BHS11 & .899 & .013 & -71.722 & $<.001$ \\
\hline BHS12 & .78 & .017 & -46.966 & $<.001$ \\
\hline $\mathrm{BHS} 13$ & -.055 & .043 & 1.263 & .206 \\
\hline BHS14 & .745 & .017 & -43.273 & $<.001$ \\
\hline BHS15 & -.798 & .016 & 49.960 & $<.001$ \\
\hline BHS16 & .861 & .015 & -58.880 & $<.001$ \\
\hline BHS17 & .89 & .013 & -70.791 & $<.001$ \\
\hline BHS18 & .725 & .017 & -42.436 & $<.001$ \\
\hline BHS19 & -.719 & .02 & 35.290 & $<.001$ \\
\hline BHS20 & .92 & .011 & -82.167 & $<.001$ \\
\hline \multicolumn{5}{|c|}{ Method Factor } \\
\hline BHS1 & .38 & .035 & 10.714 & $<.001$ \\
\hline BHS3 & .15 & .042 & 3.544 & $<.001$ \\
\hline BHS5 & .141 & .044 & 3.216 & $<.01$ \\
\hline BHS6 & .36 & .052 & 6.914 & $<.001$ \\
\hline BHS8 & -.012 & .042 & -.295 & .768 \\
\hline BHS10 & .144 & .041 & 3.501 & $<.001$ \\
\hline $\mathrm{BHS} 13$ & .353 & .057 & 6.184 & $<.001$ \\
\hline BHS15 & .486 & .038 & 12.668 & $<.001$ \\
\hline BHS19 & .218 & .038 & 5.716 & $<.001$ \\
\hline
\end{tabular}

Note. CT-C(M-1), correlated trait-correlated method minus one.

Table 1). In the ROC analysis, the AUC measures the performance of a classifier, and a higher AUC value means a better classification. The AUC can be considered an index of the discriminating ability of a test, and an AUC of 0.5 is equivalent to tossing a coin (i.e., an uninformative test). Statistical analysis was performed using the pROC package (Robin et al., 2011) in R (R Core Team, 2016).

\section{Results}

\section{Internal structure of BHS: Comparison of measurement models}

As shown in Table 2, Models 3 and 4 fit better than Model 5, and Model 5 fits better than Models 1 and 2 .

While Models 3 and 4 fit better than Model 5, they evidenced anomalous results (i.e., negative variances of specific factors and non-significant and/or incoherent negative loadings in the specific factors; Eid, 2020; Flores-Kanter et al., 2018). In other words, Models 3 and 4 are suboptimal (Heinrich et al., 2020). Based on these results, it can be concluded that the best fit is achieved by Model 5 .

Table 3 summarizes the standardized factor loadings corresponding to the M-1 model with positive words as referenced items (Model 5). Except for item 13, all items load strongly in the trait factor $(>.50)$ and in the expected direction (all $p<.001)$.

\section{Criterion-related validity}

\section{Testing the structural model}

In support of hypothesis (H1), the trait factor presents a larger $(b=.754, p<.001)$ and more significant effect than the low-magnitude effect observed in the case of the MF $(b=.201, p .008)$. However, the MF has a statistically significant effect on SI. Although the effect is low, this result may suggest the presence of a certain trait and not just a residual method effect (Geiser et al., 2008). Based on the previous CFA findings, we hypothesized that this effect could be mediated mainly by item 13 . The analyses-excluding item 13-were repeated to corroborate this. The results indicated that the trait factor has a strong effect on SI $(b=$ $.753, p<.001)$, while the method effect decreases in magnitude, not reaching statistical significance $(b=.152, p$ .052; see Figure 2).

Since relating external variables directly (i.e., direct effects) to general factors and specific factors can lead to biased parameter estimates (Koch et al., 2018), we repeated the analysis with the same SEM model, but we estimated the correlation between the trait factor and MF with SI (see Gomez \& Watson, 2017). In this case, the correlation between the trait factor and the SI remained high, while the correlation between the MF and SI had a marginal statistical significance and showed a very small or null effect. This occurred when item 13 was included (SI with MF $\mathrm{r}=.088$, $p=.009$; SI with trait factor $\mathrm{r}=.753, p<.001$ ) and when it was excluded (SI with MF r $=.068, p=.047$; SI with trait factor $\mathrm{r}=.753, p<.001$ ).

\section{ROC curve procedure}

For comparison testing, we used the comparison based on the AUC and the bootstrap percentile method for paired or 


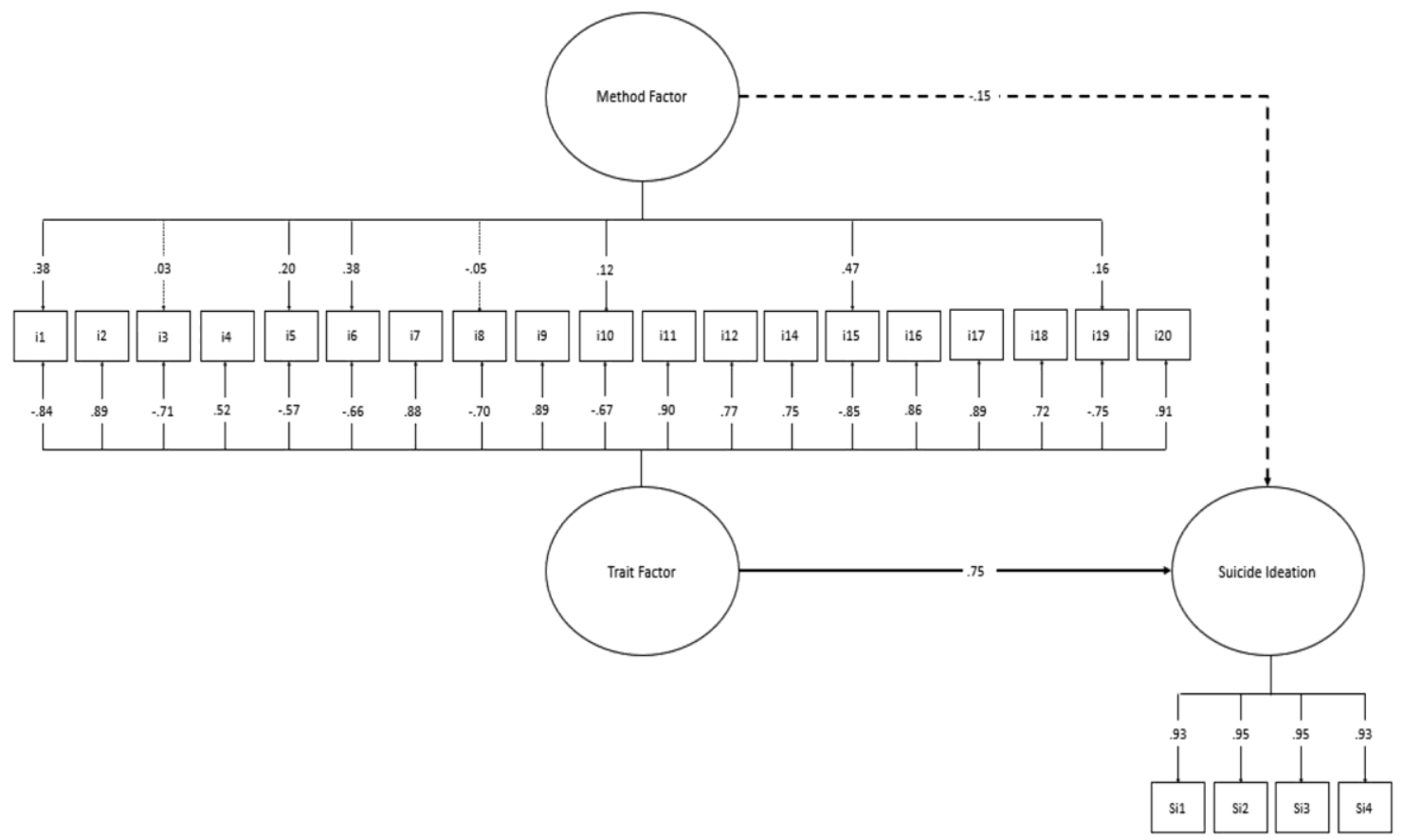

Figure 2. Structural model:Trait factor and method factor effects on suicide ideation. Standardized factor loadings. Dashed lines=statistically insignificant factor loading or regression coefficient $(p>.05)$. Solid lines = statistically significant factor loading or regression coefficient $(p<.001)$.

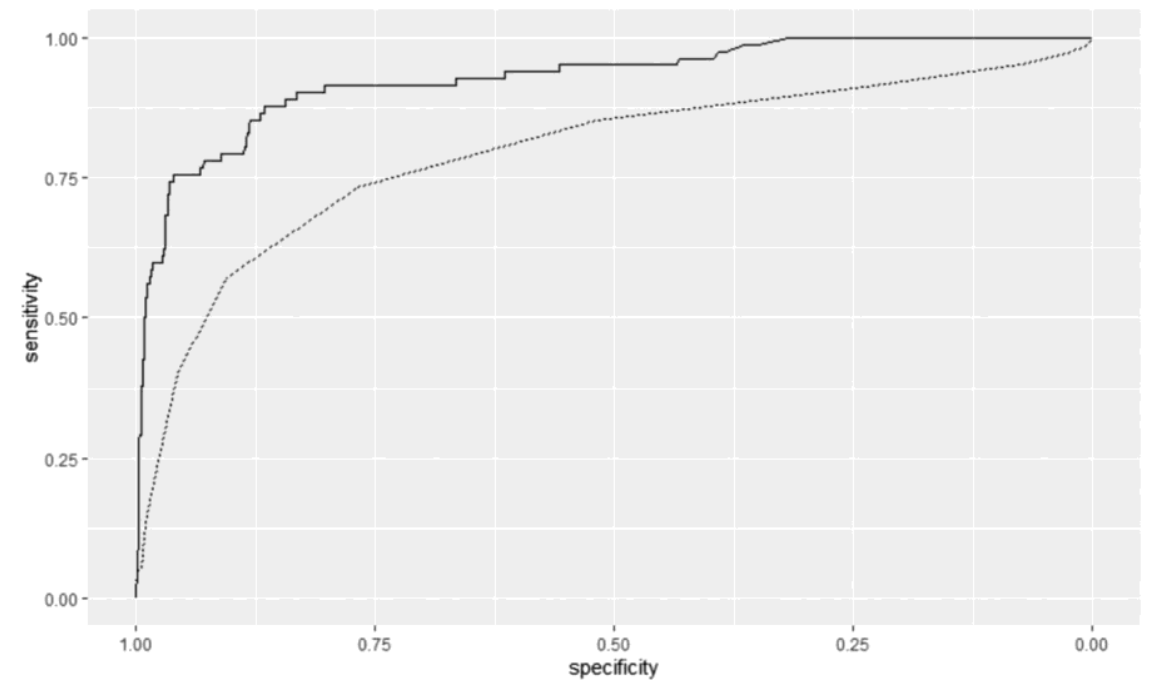

Figure 3. ROC curve. ROC curve obtained for the M-1 trait score (solid line) and BHS sum-score (dashed line).

correlated ROC curves (Robin et al., 2011). The ranges of values obtained from the factor scores derived from the M1 model and the BHS sum-score were used as a start- ing point, verifying the performance of an SI group classification. For comparative purposes, we included the total BHS sum-score due to the widespread use of this procedure in applied research. Figure 3 shows the ROC curve obtained for the trait factor score (solid line) and the BHS sum-score (dashed line). The results showed that the discrimination capacity of the factorial score obtained from the CT-C(M-1) model (AUC = .928) was significantly higher $(p<.001)$ than that observed for the BHS sumscore $($ AUC $=.794)$.

\section{Discussion}

The study's main objective was to apply a CT-C(M-1) model to analyze the internal structure of the BHS. We proposed to verify the criterion validity and relative advantage of using the factor scores derived from the M-1 model in the prediction of SI. We first hypothesized that this model would obtain better adjustment rates than those observed for previous models (H1). Furthermore, we hypothesized that the trait factor representing hopelessness for its positive items strongly explains SI (H2). By contrast, we proposed that the MF will only have a small or statistically insignificant effect beyond the effect of the trait factor (H3). Finally, we hypothesized that the discriminatory capacity of the M-1 
factor score would be higher than that observed in the previous literature, obtaining a statistically significant difference in the AUC values of the M-1 factor score compared to the BHS sum-score (H4).

Our analysis showed evidence in support of H1. The better fit obtained by the M-1 model is consistent with the initial theoretical approach on which Beck et al. (1974) based the elaboration of the scale. They pointed out that "a person's hopelessness can be objectified by defining it in terms of a system of negative expectancies concerning himself and his future life" (p. 861). In this sense, we consider that the controversies evidenced so far regarding the factor structure or construct validity of the BHS are mainly due to methodological artifacts. In this case, the trait factor could be termed pessimism measured by negatively worded items (i.e., positive items; see Geiser et al., 2008).

In the present study, it was verified that item 13 is the only MF indicator that could account for a higher-thanexpected explained variance. In much of the earlier research, this item showed problematic performance (Kliem et al., 2018). A more detailed analysis of its content shows that it is the only item that refers to the respondent's current situation and the possibility of positive change in the future. It can be hypothesized that item 13 refers to a cognitive-emotional regulation strategy of an adaptive nature, possibly associated with the refocusing or positive reinterpretation of adverse events (Flores-Kanter \& Medrano, 2020). In favor of this possibility, the structural model showed that the MF's effect does not achieve statistical significance when item 13 is subtracted. In this sense, the content validity of item 13 in the context of the BHS is debatable, and it is suggested that in future applications of the scale the results should be verified by considering the inclusion/exclusion of this item.

With respect to $\mathrm{H} 2$ and $\mathrm{H} 3$, when we applied the structural model based on the M-1 measurement, the presence of a strong effect of the trait factor on the SI was seen, compared to the MF's low and statistically insignificant impact on SI (Figure 2). This result was confirmed by verifying the correlations between the trait factor and MF with the SI. Therefore, this suggests that a trait factor (i.e., pessimism measured by negatively worded items) exerted the greatest amount of variance on a criterion variable of interest, and no substantial variance that can be explained beyond this trait factor was evident (Geiser et al., 2008).

We also obtained evidence in support of $\mathrm{H} 4$. The results of the present work show that the discrimination capacity of people with high SI and no SI obtained from the BHS sumscore is similar to that obtained in previous studies (see Gran6 et al., 2017), while the discrimination capacity of the factorial score of the trait factor obtained from the CT- C(M1) model is significantly higher. These are very import- ant results since the BHS sum-score is the major statistic interpreted in everyday practice (Kliem et al., 2018). Based on these results, we support can conclude in favor of using the factorial score derived from the CT-C(M-1), as opposed to the widespread practice of calculating the BHS sum-score for discriminatory/predictive purposes.
At this point, it is essential to make some observations regarding the use of bifactor models in psychology and related sciences. Bifactor models are increasingly used for analyses in different research areas in psychology and related sciences as one way to control the method effect (Bonify et al., 2016). In these scenarios, the objective of the analysis is to verify the reliable total score after controlling for some MFs, such as reversed or negative items (Eid et al., 2016). There is strong evidence of the method effect in studies that use other scales, one example being the Rosenberg SelfEsteem Scale. Likewise, in other scales similar to the BHS (see Maydeu-Olivares \& Coffman, 2006), the same problem has been observed due to the incorporation of reversed or negative items that show optimism or a positive vision of the future. It is possible to verify the presence of the method effect, and correct modeling has enabled an improvement in the use of the scale and its factor scores (Salerno et al., 2017). In line with these arguments, the results obtained in the present research evidenced the need for and importance of considering factor scores derived from well-fitting CFA models.

In this field, it can be difficult for researchers to choose among several methods to model this type of method-related effect. This issue may explain the frequent misuse of bifactor models (see Bonifay et al., 2016). Therefore, it is important to highlight some evidence-based suggestions to guide good practice (Flores-Kanter et al., 2018). In the case of modeling the method effect on scales similar to the self-esteem or hopelessness-pessimism scales, the CT-C(M-1) models have proven to be advantageous over other modeling possibilities (e.g., random intercepts, symmetries CT-CM; see Geiser et al., 2008; Burns et al., 2020 ). In this research, the CTCM and MTMM models showed poor specification errors that prevented their identification or the estimation of the corresponding parameters, presenting an inadequate fit. Finally, it is important to emphasize specific points related to the correct reading of the results obtained in this type of approach. In the case of the M-1 model, the key aspect is the differentiation between (a) a spurious method effect or (b) the presence of a differential feature (Marsh et al., 2010) The present results regarding the BHS allow us to conclude in support of (a).

This study has some limitations that should be noted. First, participants were asked to self-report; thus, the findings should be replicated using other measures (Bonifay et al., 2016; Clark \& Watson, 2019). For example, regarding criterion-related validity, other behavioral measures (e.g., suicide attempts) could be incorporated. Second, this study utilized a cross-sectional observational design; however, the implementation of longitudinal and repetitive measures (e.g. ecological momentary assessment; see Kim et al., 2019) would enable future researchers to make more precise inferences regarding the state-trait hopelessness construct as well as mutual relationships with relevant external variables. Finally, future research should extend these findings to other populations (e.g., clinical samples; Boduszek \& Dhingra, 2016). 


\section{Conclusions}

This study provides evidence in support of the CT-C(M-1) model for modeling the BHS internal structure, and the trait factor could, in this case, be termed pessimism measured by negatively worded items. The resulting trait factor score showed criterion validity by presenting a substantial effect on SI indicators. Further, the trait factor score evidenced better and optimal discrimination between people with high SI and no SI. In concordance with Wetzel and Roberts (2020) and Flores-Kanter (2017), we hope that the applied field does not undervalue good measurement and evidencebased research practices or ignore the measurement research that has been done.

\section{References}

Aish, A., \& Wasserman, D. (2001). Does Beck's Hopelessness Scale really measure several components? Psychological Medicine, 31(2), 367-372. https://doi.org/10.1017/S0033291701003300

American Psychological Association. (2017). Ethical principles of psychologists and code of conduct. EEUU. http://www.apa.org/ethics

Asparouhov, T., \& Muthen, B. (2006). Robust chi square difference testing with mean and variance adjusted test statistics. Mplus Web Notes: $\mathrm{N}^{\circ}$ 10. http://statmodel.com/download/webnotes/webnote10. pdf

Barredo, J., Aiken, E., van 't Wout-Frank, M., Greenberg, B. D., Carpenter, L. L., \& Philip, N. S. (2019). Neuroimaging correlates of suicidality in decision-making circuits in posttraumatic stress disorder. Frontiers in Psychiatry, 10. https://doi.org/10.3389/fpsyt.2019. 00044

Beck, A. T., Weissman, A., Lester, D., \& Trexler, L. (1974). The measurement of pessimism: The Hopelessness Scale. Journal of Consulting and Clinical Psychology, 42(6), 861-865. https://doi.org/ 10.1037/h0037562

Boduszek, D., \& Dhingra, K. (2016). Construct validity of the Beck Hopelessness Scale (BHS) among university students: A multitrait- multimethod approach. Psychological Assessment, 28(10), 1325-1330. https://doi.org/10.1037/pas0000245

Bonifay, W., Lane, S. P., \& Reise, S. P. (2016). Three concerns with applying a Bifactor Model as a structure of psychopathology. Clinical Psychological Science, 5(1), 184-186. https://doi.org/10.1177/ 2167702616657069

Brown, G. K., Beck, A. T., Steer, R. A., \& Grisham, J. R. (2000). Risk factors for suicide in psychiatric outpatients: A 20-year prospective study. Journal of Consulting and Clinical Psychology, 68(3), 371-377. https://doi.org/10.1037/0022-006x.68.3.371

Burns, G. L., Geiser, C., Servera, M., Becker, S. P., \& Beauchaine, T. P. (2020). Application of the bifactor $S-1$ model to multisource ratings of ADHD/ODD symptoms: An appropriate bifactor model for symptom ratings. Journal of Abnormal Child Psychology, 48(7), 881894. https://doi.org/10.1007/s10802-020-00629-4

Clark, L. A., \& Watson, D. (2019). Constructing validity: New developments in creating objective measuring instruments. Psychological Assessment, 31(12), 1412-1427. https://doi.org/10.1037/pas0000626

DiStefano, C., \& Motl, R. W. (2006). Further investigating method effects associated with negatively worded items on self-report surveys. Structural Equation Modeling: A Multidisciplinary Journal, 13(3), 440-464. https://doi.org/10.1207/s15328007sem1303_6

Eid, M. (2020). Multi-faceted constructs in abnormal psychology: Implications of the bifactor S-1 model for individual clinical assessment. Journal of Abnormal Child Psychology, 48(7), 895-900. https:// doi.org/10.1007/s10802-020-00624-9

Eid, M., Geiser, C., \& Koch, T. (2016). Measuring method effects. Current Directions in Psychological Science, 25(4), 275-280. https:// doi.org/10.1177/0963721416649624

Fernández-Liporace, M., \& Casullo, M. M. (2006). Validación factor de una escala para evaluar riesgo suicida [Factor validity of a scale to assess suicidal risk]. Revista Iberoamericana de Diagnóstico y Evaluación Psicológica, 21(1), 9-22.

Flora, D. B., \& Curran, P. J. (2004). An empirical evaluation of alternative methods of estimation for confirmatory factor analysis with ordinal data. Psychological Methods, 9(4), 466-491. https://doi.org/ 10.1037/1082-989X.9.4.466

Flores-Kanter, P. E. (2017). El lugar de la psicolog' $1 a$ en las investigaciones empíricas del suicidio en argentina: Un estudio Bibliométrico [State of psychological empirical research on suicide in argentine: A bibliometric study]. Interdisciplinaria: Revista de Psicolog' za y Ciencias Afines, 34(1). https://doi.org/10.16888/interd.2017.34.1.2

Flores-Kanter, P. E., \& Medrano, L. A. (2020). Commentary: Putting 'emotional intelligences' in their place: Introducing the integrated model of affect-related individual differences.Frontiers in Psychology, 11, 574. https://doi.org/10.3389/fpsyg.2020.00574

Flores-Kanter, P. E., Dominguez-Lara, S., Trógolo, M. A., \& Medrano, L. A. (2018). Best practices in the use of bifactor models: Conceptual grounds, fit indices and complementary indicators. Revista Evaluar, 18(3), 44-48. https://doi.org/10.35670/1667-4545. v18.n3.22221

Flores-Kanter, P. E., Garc' 1a-Batista, Z. E., Moretti, L. S., \& Medrano, L. A. (2019). Towards an explanatory model of suicidal ideation: The effects of cognitive emotional regulation strategies, affectivity and hopelessness. The Spanish Journal of Psychology, 22. https://doi. org/10.1017/sjp.2019.45

Geiser, C., Eid, M., \& Nussbeck, F. W. (2008). On the meaning of the latent variables in the CT-C(M-1) model: A comment on MaydeuOlivares and Coffman (2006). Psychological Methods, 13(1), 49-57. https://doi.org/10.1037/1082-989X.13.1.49

Gomez, R., \& Watson, S. D. (2017). Confirmatory factor analysis of the combined social phobia scale and social interaction anxiety scale: Support for a bifactor model. Frontiers in Psychology, 8, 70 https:// doi.org/10.3389/fpsyg. 2017.00070

G6nen, M. (2007). Analyzing receiver operating characteristic curves with SAS. SAS Publishing.

Granat, A., Gadassi, R., Gilboa-Schechtman, E., \& Feldman, R. (2017). Maternal depression and anxiety, social synchrony, and infant regulation of negative and positive emotions. Emotion (Washington D.C.), 17(1), 11-27. https://doi.org/10.1037/emo0000204 
Gran6, N., Oksanen, J., Kallionpe€6, S., \& Roine, M. (2017). Specificity and sensitivity of the Beck Hopelessness Scale for suicidal ideation among adolescents entering early intervention service. Nordic Journal of Psychiatry, 71(1), 72-76. https://doi.org/10.1080/08039488. 2016.1227370

Habibzadeh, F., Habibzadeh, P., \& Yadollahie, M. (2016). On determining the most appropriate test cut-off value: The case of tests with continuous results. Biochemia Medica, 26, 297-307. https://doi.org/ 10.11613/BM.2016.034

Heinrich, M., Zagorscak, P., Eid, M., \& Knaevelsrud, C. (2020). Giving $\mathrm{G}$ a meaning: An application of the bifactor-(S-1) approach to realize a more symptom-oriented modeling of the beck depression inventory-II. Assessment, 27(7), 1429-1447. https://doi.org/10.1177/ 1073191118803738

Hu, L., \& Bentler, P. M. (1999). Cutoff criteria for fit indexes in covariance structure analysis: Conventional criteria versus new alternatives. Structural Equation Modeling: A Multidisciplinary Journal, 6(1), 1-55. https://doi.org/10.1080/10705519909540118

Innamorati, M., Lester, D., Balsamo, M., Erbuto, D., Ricci, F., Amore, M., Girardi, P., \& Pompili, M. (2014). Factor validity of the Beck Hopelessness Scale in Italian medical patients. Journal of Psychopathology and Behavioral Assessment, 36(2), 300-307. https:// doi.org/10.1007/s10862-013-9380-3

Kim, J., Marcusson-Clavertz, D., Yoshiuchi, K., \& Smyth, J. M. (2019). Potential benefits of integrating ecological momentary assessment data into mHealth care systems. BioPsychoSocial Medicine, 13(1), 19.https://doi.org/10.1186/s13030-019-0160-5

Kliem, S., Lohmann, A., M6ßle, T., \& Brfhler, E. (2018). Psychometric properties and measurement invariance of the Beck hopelessness scale (BHS): Results from a German representative population sample. BMC Psychiatry, 18(1). https://doi.org/http://dx.doi.org/10.1186/ s12888-018-1646-6

Kocalevent, R.-D., Finck, C., Pérez-Trujillo, M., Sautier, L., Zill, J., \& Hinz, A. (2017). Standardization of the Beck Hopelessness Scale in the general population. Journal of Mental Health, 26(6), 516-522. https://doi.org/10.1080/09638237.2016.1244717

Koch, T., Kelava, A., \& Eid, M. (2018). Analyzing different types of moderated method effects in confirmatory factor models for structurally different methods. Structural Equation Modeling: A Multidisciplinary Journal, 25(2), 179-200. https://doi.org/10.1080/ 10705511.2017.1373595

Marsh, H. W., Hau, K. T., \& Wen, Z. (2004). In search of golden rules: Comment on hypothesis-testing approaches to setting cutoff values for fit indexes and dangers in overgeneralizing $\mathrm{Hu}$ and Bentler's (1999) findings. Structural Equation Modeling: A Multidisciplinary Journal, 11(3), 320-341. https://doi.org/10.1207/s15328007sem1103_2

Marsh, H. W., Leldtke, O., Muthén, B., Asparouhov, T., Morin, A. J. S., Trautwein, U., \& Nagengast, B. (2010). A new look at the big five factor structure through exploratory structural equation modeling. Psychological Assessment, 22(3), 471-491. https://doi.org/10.1037/ a0019227.supp

Maydeu-Olivares, A., \& Coffman, D. L. (2006). Random intercept item factor analysis. Psychological Methods, 11(4), 344-362. https://doi. org/10.1037/1082-989X.11.4.344

Mikulic, I. M., Casullo, G. L., Crespi, M. C., \& Marconi, A. (2009). Escala de Desesperanza BHS (A. Beck, 1974): Estudio de las propiedades psicométricas y baremización de la Adaptación Argentina [Beck Hopelessness Scale BHS (A. BECK, 1974): Psychometric study and standardization of Argentinian adaptation]. Anuario de Investigaciones, 16, 365-373.

R Core Team. (2016). R: A language and environment for statistical computing. R Foundation for Statistical Computing.

Rhemtulla, M., Brosseau-Liard, P. É., \& Savalei, V. (2012). When can categorical variables be treated as continuous? A comparison of robust continuous and categorical SEM estimation methods under suboptimal conditions. Psychological Methods, 17(3), 354-373. https://doi.org/10.1037/a0029315

Robin, X., Turck, N., Hainard, A., Tiberti, N., Lisacek, F., Sanchez, J.C., \& MEller, M. (2011). pROC: An open-source package for R and
S po analyze and compare ROC curves. BMC Bioinformatics, 12(1). https://doi.org/10.1186/1471-2105-12-77

Salerno, L., Ingoglia, S., \& Lo Coco, G. (2017). Competing factor structures of the Rosenberg Self-Esteem Scale (RSES) and its measurement invariance across clinical and non-clinical samples. Personality and Individual Differences, 113, 13-19. https://doi.org/10.1016/j.paid. 2017.02.063

Savalei, V., \& Falk, C. F. (2014). Recovering substantive factor loadings in the presence of acquiescence bias: A comparison of three approaches. Multivariate Behavioral Research, 49(5), 407-424. https://doi.org/10.1080/00273171.2014.931800

Serafini, G., Lamis, D. A., Aguglia, A., Amerio, A., Nebbia, J., Geoffroy, P. A., ... Amore, M. (2020). Hopelessness and its correlates with clinical outcomes in an outpatient setting. Journal of Affective Disorders, 263, 472-479. https://doi.org/10.1016/j.jad.2019.11.144

Szabó, M., Mészáros, V., Sallay, J., Ajtay, G., Boross, V., UdvardyMészáros, À., Vizin, G., \& Perczel-Forintos, D. (2016). The beck hopelessness scale. specific factors of method effects? European

Journal of Psychological Assessment, 32(2), 111-118. https://doi.org/ 10.1027/1015-5759/a000240

The International Test Commission. (2006). International guidelines on computer-based and internet-delivered testing. International Journal of Testing, 6(2), 143-171.https://doi.org/10.1207/s15327574ijt0602_4

Tomas, J. M., \& Oliver, A. (1999). Rosenberg's self-esteem scale: Two factors or method effects. Structural Equation Modeling: A Multidisciplinary Journal, 6(1), 84-98. https://doi.org/10.1080/ 10705519909540120

Tsujii, N., Shirakawa, O., Niwa, A., Yonemoto, N., Kawanishi, C., Yamamoto, K., Sugimoto, T., \& Hirayasu, Y. (2020). Hopelessness is associated with repeated suicidal behaviors after discharge in patients admitted to emergency departments for attempted suicide. Journal of Affective Disorders, 272, 170-175. https://doi.org/10.1016/ j.jad.2020.04.037

Vecco, G. A., Flores-Kanter, P. E., \& Luque, L. E. (2021). Análisis psicométrico del inventario de orientación suicida ISO-19, en adolescentes cordobeses escolarizados [Psychometric analysis of the Inventory of Suicide Orientation ISO-19, in scholarized adolescents]. Revista Evaluar, 21(1), 39-52. https://doi.org/10.35670/1667-4545. v21.n1.32831

Weigold, A., Weigold, I. K., \& Russell, E. J. (2013). Examination of the equivalence of self-report survey-based paper-and-pencil and internet data collection methods. Psychological Methods, 18(1), 53-70. https://doi.org/10.1037/a0031607

Wenzel, A., Brown, G. K., \& Beck, A. T. (2009). Cognitive therapy for suicidal patiens. Scientific and clinical applications. American Psychological Association.

Wetherall, K., Cleare, S., Eschle, S., Ferguson, E., O'Connor, D. B., O'Carroll, R. E., \& O'Connor, R. C. (2018). From ideation to action: Differentiating between those who think about suicide and those who attempt suicide in a national study of young adults. Journal of Affective Disorders, 241, 475-483. https://doi.org/10.1016/j.jad.2018. 07.074

Wetzel, E., \& Roberts, B. W. (2020). Commentary on Hussey and Hughes (2020): Hidden Invalidity Among 15 Commonly Used Measures in Social and Personality Psychology. Advances in Methods and Practices in Psychological Science, 3(4), 505-508. https://doi.org/ $10.1177 / 2515245920957618$.

Yuan, K. H., \& Bentler, P. M. (2007). Structural equation modeling. In C. R. Rao \& S. Sinharay (Eds.), Handbook of statistics, Volume 26: Psychometrics (pp. 297-358). North-Holland.

Zhang, X., \& Savalei, V. (2016). Improving the factor structure of psychological scales: The expanded format as an alternative to the likert scale format. Educational and Psychological Measurement, 76(3), 357-386. https://doi.org/10.1177/0013164415596421

Zhu, Z., Liu, Y., Zhang, C., Yuan, Z., Zhang, Q., Tang, F., Lin, H., Zhang, Y., Liu, L., \& Xue, F. (2013). Identification of cardiovascular risk components in urban Chinese with metabolic syndrome and application to coronary heart disease prediction: A longitudinal study. PLoS One, 8(12), e84204. https://doi.org/10.1371/journal.pone. 0084204 